DE

M E D I C I N A

T R O P I C A L

$\mathrm{DE}$

SÃO PAULO

JOURNAL OF THE SÃO PAULO INSTITUTE OF TROPICAL MEDICINE

${ }^{1}$ University of Belgrade, Faculty of Medicine, Belgrade, Serbia

${ }^{2}$ Clinical Centre of Serbia, Clinic for Pulmonology, Belgrade, Serbia

${ }^{3}$ University of St. Andrews, School of Medicine, St Andrews, United Kingdom

${ }^{4}$ Clinical Centre of Serbia, Clinic for Infectious and Tropical Diseases, Belgrade, Serbia

${ }^{5}$ Clinical Centre of Serbia, Clinic for Gynecology and Obstetrics, Belgrade, Serbia

${ }^{6}$ University of Sassari, Department of Biomedical Sciences, Sassari, Italy

Correspondence to: Aleksandra Barac Clinical Centre of Serbia, Clinic for Infectious and Tropical Diseases, Bul. Oslobodjenja 16, Belgrade, Serbia Tel: +38 1631869502

E-mail: aleksandrabarac85@gmail.com

Received: 13 September 2019

Accepted: 4 December 2019

\section{An exceptional case report of disseminated cryptococcosis in a hitherto immunocompetent patient}

\author{
Tatjana Adzic-Vukicevic ${ }^{1,2}$, Muge Cevik ${ }^{3}$, Jasmina Poluga ${ }^{1,4}$, Jelena Micic ${ }^{1,5}$, \\ Salvatore Rubino ${ }^{6}$, Bianca Paglietti ${ }^{6}$, Aleksandra Barac ${ }^{1,4}$
}

\section{ABSTRACT}

Cryptococcosis is an opportunistic fungal infection causes significant disease predominantly in immunocompromised patients. Here we present an excepcional case of disseminated cryptococcosis with pulmonary and cerebral involvement in an immunocompetent patient with no apparent predisposing factors at the time of hospital admission. We described a case of an apparently immunocompetent 66-years old man admitted to hospital with a one-month history of cough, fever and vertigo. During hospitalization, thorax imaging was suggestive of lung metastasis, therefore, he went through several investigations. During hospitalization, he developed neurological symptoms and subsequently underwent a lumbar puncture. Cerebrospinal fluid (CSF) culture was positive for Cryptococcus spp. isolated on Sabouraud's dextrose agar and bird seed agar. In addition, the direct microscopy examination was positive for the India ink test, as well as with the latex agglutination test for cryptococcal polysaccharide antigen $(\mathrm{CrAg})$ in $\mathrm{CSF}$, while serum $\mathrm{CrAg}$ was negative. Despite the absence of classic immunocompromising features, he was treated with amphotericin B and fluconazole due to suspected disseminated cryptococcal infection. Later, he was diagnosed with prostatic adenocarcinoma. Upon successful completion of treatment for disseminated cryptococcosis, the patient underwent radical prostate ablation surgery as a treatment forprostatic adenocarcinoma. This exceptional case emphasizes the high degree of suspicion of atypical infections, and in these cases, it is particularly important to consider fungal infections in hitherto healthy patients with no apparent predisposing factors. Although Cryptococcus spp. is predominantly reported in patients with hematological malignancies, cryptococcosis investigation should also be considered as part of the initial workup of patients with a new diagnosis of a solid tumour prior to chemotherapy or radiotherapy.

KEYWORDS: Cryptococcus. Disseminated infection. Malignancy. Prostatic adenocarcinoma.

\section{INTRODUCTION}

Cryptococcosis is an opportunistic fungal infection that has been historically regarded as a disease of immunocompromised patients. Cryptococcus is an ubiquitous yeast, and the most common species causing disease are Cryptococcus neoformans and C. gatii ${ }^{1}$. Cryptococcus neoformans is the predominant cause of cryptococcosis among people living with the human immunodeficiency virus (HIV), but it can also be found in other immunocompromised hosts such as solid organ transplant recipients, patients on prolonged glucocorticoid therapy, hematologic malignancies and in those with impaired cell-mediated immunity ${ }^{2,3}$.

Although disseminated cryptococcosis is a rare condition that almost always occurs in immunocompromised hosts especially in persons living with $\mathrm{HIV}^{4}$, there 
is growing evidence suggesting that disseminated disease may also occur in otherwise healthy, HIV-uninfected, nontransplant patients in whom an underlying predisposing factor may not be apparent ${ }^{5,6}$. Here we present an exceptional case report of disseminated cryptococcosis with pulmonary and cerebral involvement in an apparently immunocompetent patient with no apparent predisposing factors at the time of hospital admission.

\section{CASE REPORT}

A previously healthy 66-years old man was admitted to hospital with a one-month history of cough, fever and vertigo, being initially treated with macrolides and cephalosporins for 3 weeks in the community with an unsuccessful outcome. He had a past medical history of myocardial infarction and revascularization through stent insertion one year prior to hospital admission. He worked as an engineer in South-West Africa for 6 years, without previous exposure to pigeons. At the time of admission, he was hemodynamically stable with a blood pressure of $130 / 70 \mathrm{~mm} / \mathrm{Hg}$, heart rate of $90 \mathrm{bpm}$, oxygen saturation of $96 \%$ in room air, although he was tachypneic (respiratory rate of $25 \mathrm{bpm}$ ) and had temperature of $37.8^{\circ} \mathrm{C}$. Chest examination revelated generalized crepitations in both lungs, cardiac auscultation was normal, abdomen was soft and non-tender with no organ enlargement and neurological examination was unremarkable. Laboratory findings showed high erythrocyte sedimentation rate of $88.0 \mathrm{~mm} / \mathrm{h}$ (normal range $0-20 \mathrm{~mm} / \mathrm{h}$ ), C-reactive protein (CRP) $85.2 \mathrm{mg} / \mathrm{L}$ (normal range $0-5 \mathrm{mg} / \mathrm{L}$ ) and white blood count $19,500 / \mu \mathrm{L}(4,000-10,000 / \mu \mathrm{L})$ with neutrophilia of $83.3 \%$. Other biochemistry and hematology results were unremarkable. Electrocardiogram showed left bundle branch block with occasional ventricular arrhythmias.

The chest X-ray showed bilateral pulmonary opacities (Figure 1A). Subsequently, the patient underwent thorax computed tomography (CT) scan that revealed multiple bilateral patchy opacities with particular nodular excavations with no evidence of mediastinal lymph node enlargement (Figure 1B). Due to the pathological images seen in the lungs, the patient received azithromycin 1 x $500 \mathrm{mg}$ BD i.v. and ceftriaxone $2 \times 1 \mathrm{~g}$ OD i.v. Despite the antibiotic treatment, the follow-up chest X-ray showed persistence of abnormal images, raising the suspicion of lung metastases.

$\mathrm{He}$ then underwent multiple microbiological investigations including sputum smear, sputum culture, blood cultures, blood borne viruses screening (hepatitis B, hepatitis C and HIV) and serology for Legionella sp., Mycoplasma sp., Histoplasma capsulatum, Coccidioides sp., Candida sp. and Aspergillus sp., that were all negative. Subsequently, fiberoptic bronchoscopy was performed with transbronchial biopsy. Histopathological findings of the lung tissue were suggestive of non-specific chronic bronchitis or peribronchitis. The bronchoalveolar lavage (BAL) culture was negative for bacterial and fungal pathogens as well as Mycobacterium sp. Arterial blood gas analysis was normal and lung function testing were within normal range with FVC of $102 \%$ and FEV1 of $99 \%$. QuantiFERON-TB gold test was also negative.

Later during hospitalization, the patient developed headache with worsening of vertigo and fever. Therefore, endocranial contrasted CT scan was performed and did not reveal any metastasis or acute brain hemorrhage. Subsequently, lumbar puncture was performed to rule out an infectious or malignant cause of central nervous system involvement. The cerebrospinal fluid (CSF) results revealed an increased opening pressure $\left(150 \mathrm{~cm} / \mathrm{H}_{2} \mathrm{O}\right.$ ) (normal range $10-20 \mathrm{~cm} / \mathrm{H}_{2} \mathrm{O}$ ), pleocytosis with predominance of neutrophils $\left(122 / \mathrm{mm}^{3} ; 90 \%\right.$ neutrophils and $10 \%$ lymphocytes), hyperproteinorrachia $(121 \mathrm{mg} / \mathrm{dL})$ and hypoglycorrhachia (CSF glycorrhachia/plasmaglucose: $1.7 / 4.2 \mathrm{mmol} / \mathrm{L}$ ). The cerebrospinal fluid (CSF) culture was positive for Cryptococcus spp. in Sabouraud's dextrose
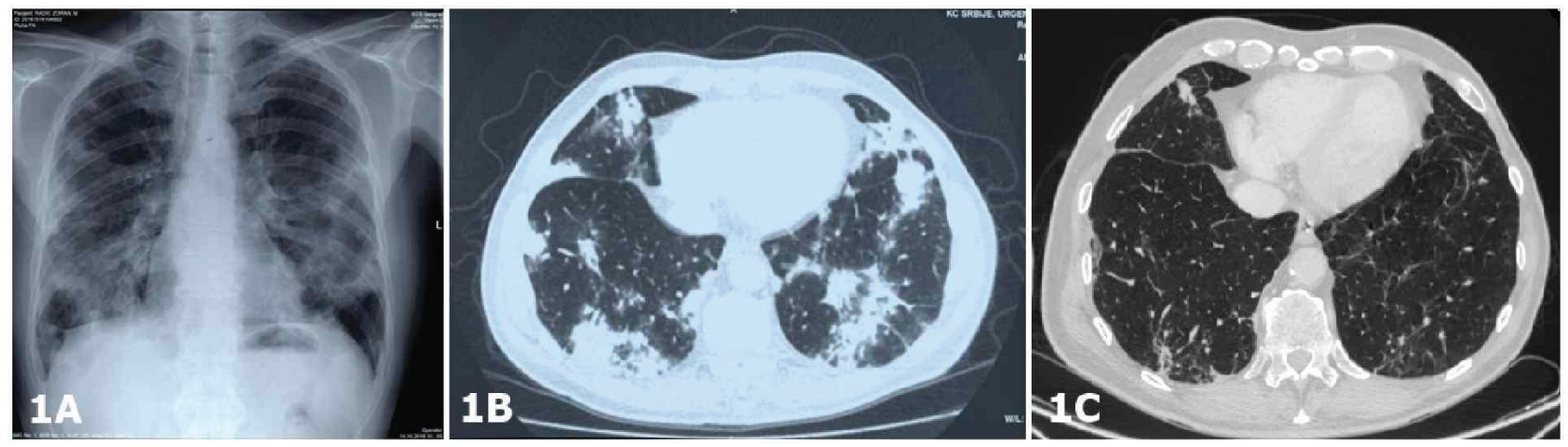

Figure 1 - A) Chest X-ray showing bilateral pulmonary opacities; B) Initial thoracic CT scan showing multiple bilateral patchy opacities with nodular excavations; C) Follow-up thoracic CT scan performed 2-months after the antifungal treatment showing a significant improvement with remaining fibrotic changes. 
agar; in addition to direct microscopy examination that was positive for India ink test as well as latex agglutination test for cryptococcal polysaccharide antigen $(\mathrm{CrAg})$ in $\mathrm{CSF}$ (titer 1:32), while serum $\mathrm{CrAg}$ was negative. The isolate was identified as Cryptococcus spp. using bird seed agar, a selective medium for Cryptococcus spp. differential isolation (Staib's medium, HiMedia, India). The brown pigmented colonies of Cryptococcus spp. grew after 2 days at $37^{\circ} \mathrm{C}$. Despite the absence of classic immunocompromising features, i.v. amphotericin $\mathrm{B}(\mathrm{AmB})$ deoxycholate formulation $(1 \mathrm{mg} / \mathrm{kg} /$ day $)$ in combination with oral fluconazole $(800 \mathrm{mg} /$ day) was initiated for suspected disseminated cryptococcosis.

Although he had no red flag symptoms indicative of malignancy, he had a complete panel of tests including tumor markers which were all within normal ranges except for the prostatic specific antigen (PSA) which was $26 \mathrm{ng} / \mathrm{mL}$ (normal range $0-4.5 \mathrm{ng} / \mathrm{mL}$ ). Given the increased PSA levels, a trans-urethral prostatic biopsy was performed, and the diagnosis of prostatic adenocarcinoma was confirmed.

After 7 days of treatment, the patient developed acute renal injury; therefore, i.v. AmB was interrupted and oral fluconazole $(800 \mathrm{mg} / \mathrm{day})$ was continued. The follow-up CSF after 4 weeks of high dose oral fluconazole treatment revealed significant improvement (2 lymphocytes, glycorrhachia $3.0 \mathrm{mmol} / \mathrm{L}$, proteinorrhachia $35 \mathrm{mg} / \mathrm{dL}$ ), while the CrAg titer in CSF was 1:8. He then continued on $400 \mathrm{mg} /$ day oral fluconazole to complete 6 months therapy in total. Diagnostic follow-up after 3 months therapy showed negative CrAg in CSF. The follow-up thoracic CT scan 2 months after treatment showed significant improvement with remaining fibrotic changes (Figure 1C).

Upon successful completion of treatment for disseminated cryptococcosis, the patient went a radical ablation surgery for prostatic adenocarcinoma with no requirement for additional cancer treatment. According to the follow-up visit 1 year after surgery and 1.5 years after fluconazole treatment, the patient recovered well, with no sequelae remaining.

\section{DISCUSSION}

This exceptional case report emphasises the challenges of recognising opportunistic infections in hitherto healthy, HIV-negative, non-transplanted patients with no apparent predisposing factors. Although Cryptococcus spp. is predominantly reported in patients with haematological malignancies ${ }^{3}$, this case indicates that cryptococcosis should also be considered as part of the differential diagnosis of patients with solid tumours, especially prior to chemotherapy or radiotherapy.
The respiratory tract acts as the primary portal of entry for Cryptococcus spp. ${ }^{1}$. As observed in this case, yeasts may remain dormant in subpleural spaces for years after inhalation, and fungal reactivation will occur when local immunity is suppressed ${ }^{1}$. Pulmonary cryptococcosis is usually asymptomatic or mildly symptomatic and tends to resolve spontaneously. However, in the immunocompromised host, pulmonary disease is usually symptomatic and can rapidly progress to acute respiratory distress syndrome ${ }^{5}$. After pulmonary cryptococcosis is established, the infection can spread to the central nervous system (CNS) resulting in meningitis, meningoencephalitis and disseminated infection ${ }^{4}$. Disseminated cryptococcosis in immunocompetent patients is uncommon, usually atypical and often late diagnosed ${ }^{5,6}$. CNS involvement in immunocompetent patients is particularly rare $^{2,5}$ so that lumbar puncture is not always warranted. However, if an apparently immunocompetent patient has CNS symptoms, or a high $\mathrm{CrAg}$ titer $(>1: 8)$ regardless of any CNS symptoms, a lumbar puncture is recommended to evaluate the CNS involvement ${ }^{7}$.

Disseminated cryptococcosis is diagnosed by positive culture obtained from at least two distinct sites, or by positive blood culture ${ }^{4}$. Diagnosis of pulmonary cryptococcosis involves a combination of chest imaging, sputum or BAL fungal cultures, serum $\mathrm{CrAg}$ and tissue histology. In this case, the patient had nodular chest X-ray and $\mathrm{CT}$ changes, but BAL, sputum cultures and serum $\mathrm{CrAg}$ were negative. Although $\mathrm{CrAg}$ is a rather sensitive method (up to 100\%) in people living with HIV, it is less reliable in immunocompetent patients ${ }^{5}$. According to previous studies, in immunocompetent populations, $\mathrm{CrAg}$ has a sensitivity of $56 \%$ in pulmonary disease and $86 \%$ in CNS disease 5 . In the present case, Cryptococcus spp. was only observed in CSF, and although the pulmonary cryptococcosis was not microbiologically confirmed, resolution of CT changes after treatment supports the pulmonary involvement. We can conclude that diagnosis could be made by positive blood or CSF culture or positive culture of other sites, according to the clinical manifestation, not requiring a positive culture in at least two sites, as one deep site is enough to confirm the diagnosis. Although the prostate gland is not an unusual site of cryptococcal infection and has been recognized as a reservoir of disseminated disease, we were not able to confirm the involvement of the prostate in this case.

The radiological manifestations of cryptococcosis can be atypical among immunocompetent patients, sometimes mimicking lung cancer or lung metastasis ${ }^{8,9}$. In a study by Song et al. among 23 HIV-negative patients with pulmonary cryptococcosis, nine were diagnosed with tuberculosis and six with metastatic lung cancer ${ }^{10}$. The most common radiological manifestations of pulmonary cryptococcosis 
are single or multiple noncalcified nodules, and pulmonary infiltrates, although consolidation, reticulonodular pattern and lobar opacities can also occur. Associated features may also include cavitation, lymphadenopathy and pleural effusion, frequently found in immunocompromised patients ${ }^{7}$. In the current case, multiple bilateral nodular changes were observed in thoracic CT scans as well as progressive multifocal consolidation. The lack of improvement despite antibiotic treatment and the newly diagnosed prostate carcinoma raised the suspicion of lung metastasis in this case. In cases of extensive or atypical radiological findings suggestive of malignancy or pneumonia, atypical causes of pneumonia should be investigated even in cases with high suspicion of malignancy.

Guidelines recommend oral fluconazole $400 \mathrm{mg} / \mathrm{day}$ for 6-12 months for the treatment of mild to moderate pulmonary cryptococcosis ${ }^{7}$. Severe pulmonary cryptococcosis should be treated similarly to CNS disease, including deoxycholate $\mathrm{AmB}$ $1 \mathrm{mg} / \mathrm{kg} /$ day in combination with oral fluconazole $800 \mathrm{mg} /$ day for the first 4 weeks, followed by oral fluconazole $400 \mathrm{mg} / \mathrm{day}$ for 6-12 months. There are no recommendations for the treatment of asymptomatic, single site infection (pulmonary cryptococcosis) as radiological recovery occurs regardless of antifungal therapy. In hitherto immunocompetent patients, there are some recommendations for prolonged intensive treatment based on poor outcomes and higher mortality ${ }^{5}$. However, in a subset of patients with favourable prognostic factors, a shorter but intensive treatment to 2 weeks may be used. Surgery is recommended only if symptoms persist despite antifungal therapy ${ }^{7}$.

\section{CONCLUSION}

In conclusion, this case emphasizes the high degree of suspicion of atypical infections, especially of fungal infections, in hitherto healthy patients with no apparent predisposing factors and should be considered in the differential diagnosis of patients diagnosed with solid tumors, especially prior to chemotherapy or radiotherapy.

\section{ACKNOWLEDGMENTS}

Aleksandra Barac received a research grant from the Ministry of Education, Science and Technology of the Republic of Serbia ( $\mathrm{N}^{\circ}$ III 45005$)$.

\section{AUTHORS' CONTRIBUTIONS}

$\mathrm{MC}$ and $\mathrm{AB}$ conceptualised this paper; TA, JM and $\mathrm{JP}$ were responsible for the patient management and data collection; MC, SR and AB drafted the first version of the manuscript, and all the authors provided critical feedback and contributed to the manuscript writing.

\section{CONFLICT OF INTERESTS}

All the authors declare that there is no conflict of interest.

\section{CONSENT FOR PUBLICATION}

The patient presented in this case report provided a written and signed informed consent.

\section{FUNDING}

No funding received for this work.

\section{REFERENCES}

1. Baddley JW, Dismukes WE. Cryptococcosis. In: Kauffman CA, Pappas PG, Sobel JD, Dismukers WE, editors. Essentials of clinical mycology. $2^{\text {nd }}$ ed. New York: Springer; 2011. p.207-26.

2. Pappas P. Cryptococcal infections in non-HIV-infected patients. Trans Am Climatol Assoc. 2013;124:61-79.

3. Schmazle SA, Buchwald UK, Gilliam BL, Riedel DI. Cryptococcus neoformans infection in malignancy. Mycoses. 2016;59:542-52.

4. Martin-Blondel G, Ysebaert L. Images in clinical medicine. Disseminated cryptococcosis. N Engl J Med. 2014;370:1741.

5. Bratton E, El Husseini N, Chastain CA, Lee MS, Poole C, Stürmer $\mathrm{T}$, et al. Comparison and temporal trends of three groups with Cryptococcosis: HIV-infected, solid organ transplant, and HIV-negative/non-transplant. PLoS One. 2012;7:e43582.

6. La Hoz RM, Pappas PG. Cryptococcal infection: changing epidemiology and implications for therapy. Drugs. 2013;73:495-504.

7. Perfect JR, Dismukes WE, Dromer F, Goldman DL, Graybill JR, Hamill RJ, et al. Clinical practice guidelines for the management of cryptococcal disease: 2010 update by the Infectious Disease Society of America. Clin Infect Dis. 2010;50:291-322.

8. Fox DL, Muller NL. Pulmonary cryptococcosis in immunocompetent patients: CT findings in 12 patients. AJR Am J Roentgenol. 2005;185:622-6.

9. Kim YS, Lee IH, Kim HS, Jin SS, Lee JH, Kim SK, et al. Pulmonary cryptococcosis mimicking primary lung cancer with multiple lung metastases. Tuberc Respir Dis (Seoul). 2012;73:182-6.

10. Song KD, Lee KS, Chung MP, Kwon OJ, Kim TS, Yi CA, et al. Pulmonary cryptococcosis: imaging findings in 23 non-AIDS patients. Korean J Radiol. 2010;11:407-16. 